ФГБУ «Национальный медицинский исследовательский центр им. В.А. Алмазова» Минздрава России, Санкт-Петербург, Россия

Обоснование: По данным литературы остеопения широко распространена, поэтому выявление факторов риска и определение групп своевременной профилактики остеопороза и восполнения минеральной плотности кости (МПК) актуально в настоящее время.

Цель: Изучить факторы, ассоциированные с развитием остеопении в послеродовом периоде

Memoды: Одномоментное обследование для выявления факторов развития остеопении у 112 родильниц 20-35 лет на 3-5 сутки после родов. Для оценки факторов снижения МПК проводились исследования анамнеза жизни, образа жизни, питания, антропометрических данных, акушерско-гинекологического анамнеза, течения беременности. Всем родильницам определялись уровни 25-гидроксикальциферола (25-OH-D) сыворотки крови, паратиреиоидный гормон (ПТГ). Оценка МПК проводилась методом двухэнергетической рентгеновской остеоденситометрии в трех отделах скелета. За снижение МПК, соответствующее остеопении принимали Z-критерий от -1 до -2,5 SD, ниже -2,5 SD соответствующее остеопорозу.

Результаты: По результатам рентгеновской остеоденситометрии сформировано две группы: 1 (n=70) - родильницы с остеопенией, 2 ( $n=42)$ - родильницы с нормальной МПК. В первой группе остеопения в дистальном отделе предплечья встречалась у 48\%, в поясничном отделе позвоночника - у 16\%, в проксимальном отделе бедра - у 36\% обследованных. Установлено влияние следующих возможных факторов в 1 группе пациенток: ИМТ в 15-20 лет

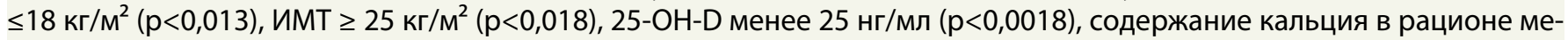
нее 800 мг/сут ( $<<0,041)$. Обнаружено влияние нарушений менструального цикла $(p<0,052)$ и преэклапсии $(p<0,042)$ на МПК в поясничном отделе позвоночника и. Дефицит витамина D выявлен у 82\% родильниц в 1 группе, у 18\% - недостаточность; во 2 группе дефицит витамина D встречался у 16\%, недостаточность - у 70\%, у 14\% - нормальные значения. У женщин с сочетанием таких факторов, как ИМТ $\leq 18$ кг/м; питание с содержанием кальция меньше 800 мг/сутки, нарушением менструального цикла в сочетании с недостаточностью витаминам D - остеопения в дистальном отделе предплечья встречалась в 11 раз чаще, чем у женщин из группы без вышеуказанных факторов (OR=11,47059; Cl $95 \%=[4,0326 ; 32,627])$.

Заключение: Наиболее существенное влияние на развитие остеопении у родильниц оказывают следующие факторы: ИМТ $\leq 18$ кг/м²; 25-OH-D<25 нг/мл; питание с содержанием кальция <800 мг/сутки, преэклампсия. Совокупность этих факторов может повышать риск развития остеопении в дистальном отделе предплечья.

КЛЮЧЕВЫЕ СЛОВА: Беременность; минеральная плотность костной ткани; витамин D; факторы риска

\title{
FACTORS INFLUENCING BONE MINERAL DENSITY IN POSTPARTUM WOMEN
}

\author{
(c) Tatiana V. Novikova, Liubov V. Kuznetsova, Natalia Yu Yakovleva, Irina E. Zazerskaya
}

Almazov National Medical Research Centre , Saint Petersburg, Russia

Background: Osteopenia is a common condition. Therefore, identification of groups for prevention of osteoporosis and restoration of bone mineral density (BMD) remains relevant.

Aim: to assess the factors contributing to development of osteopenia in puerperas.

Methods: prospective cross-sectional study. We examined 112 patients aged 20-35, 3-5 days after delivery. To assess possible factors for BMD decrease, we analyzed medical history, lifestyle, nutrition, anthropometric data, obstetric and gynecological history, and pregnancy course. We also assessed serum levels of 25-hydroxycalciferol (25-OH-D) and PTH. BMD was measured by dual energy x-ray osteodensitometry. We considered Z-score from -1 to $-2.5 \mathrm{SD}$ as osteopenia, below $-2.5 \mathrm{SD}$-as osteoporosis.

Results: based on Z-score values, two groups were formed: $1(n=70)$ - puerperas with osteopenia, 2 ( $n=42)$ - puerperas with normal BMD. In the first group, osteopenia in the distal radius was observed in $48 \%$, in the lumbar spine in $16 \%$ and in the proximal femur in 36\%. Influence of the following possible factors in group 1 was established: BMl in 15-20 years $\leq 18 \mathrm{~kg} / \mathrm{m}^{2}(\mathrm{p}<0.013), \mathrm{BMI} \geq 25 \mathrm{~kg} / \mathrm{m}^{2}(\mathrm{p}<0.018), 25-\mathrm{OH}-\mathrm{D}$ less than $25 \mathrm{ng} / \mathrm{ml}(\mathrm{p}<0.0018)$, calcium intake less than $800 \mathrm{mg} /$ day $(p<0.041)$. Menstrual disorders $(p<0.052)$ and preeclapsia $(p<0.042)$ affected lumbar spine BMD. In group 1, vitamin $D$ deficiency was detected in $82 \%$ of women, $18 \%$ showed vitamin D insufficiency; in group 2, vitamin D deficiency was found in $16 \%$, deficiency in $70 \%$, in $14 \%$ vitamin D was normal. In women with a combination of factors such as BMI $\leq 18 \mathrm{~kg} / \mathrm{m}$; calcium intake lower than $800 \mathrm{mg} /$ day, menstrual cycle disorders, vitamin D deficiency - osteopenia in the distal radius occured 
11 times more often $(\mathrm{OR}=11,47059 ; \mathrm{Cl} 95 \%=[4,0326 ; 32,627])$.

Conclusion: most significant impact on BMD decrease in puerperas can be expected if patient has the following risk factors: $\mathrm{BMI} \leq 18 \mathrm{~kg} / \mathrm{m}^{2} ; 25-\mathrm{OH}-\mathrm{D}<25 \mathrm{ng} / \mathrm{ml}$; nutrition with calcium intake $<800 \mathrm{mg}$ per day, preeclampsia. Combination of these factors may increase the risk of osteopenia in the distal radius.

KEYWORDS: Pregnancy; bone mineral density; vitamin D; risk factors

\section{ОБОСНОВАНИЕ}

Минеральная плотность костной ткани (МПК) является значимым предиктором риска переломов. Индивидуальный уровень МПК у взрослых людей является результатом между пиковой костной массой, достигнутой во время роста и последующей потерей кости в более поздние периоды жизни женщины [1,2].

Так, факторы, влияющие на костный обмен, могут способствовать недостаточному формированию пика костной массы, что впоследствии приводит к выраженному снижению МПК. Изучаются причины нарушения костного обмена у женщин молодого возраста, связанных с неполноценным питанием, курением, дефицитом массы тела, нарушением функции яичников $[3,4]$. Особенно следует выделить период беременности и лактации. Физиологическая беременность сопровождается ускорением кальциево-фосфорного обмена и метаболизма витамина-D, особенно в 3 триместре, когда происходит интенсивная минерализация скелета плода. Адаптационные механизмы включают в первую очередь повышенную абсорбцию кальция в кишечнике, за счет синтеза кальбиндина, снижение экскреции кальция почками, синтез активных метаболитов витамина D плацентарной тканью. При достаточном поступлении витамина D и кальция и адекватном уровне МПК до наступления беременности и во время гестации, указанные механизмы обеспечивают сохранение МПК [4,5]. Однако существование факторов риска снижения МПК до беременности и недостаточное питание могут приводить к повышенной резорбция кальция из органического матрикса трабекулярных костей. Для такой категории женщин беременность и лактация будут значимым фактором риска снижения МПК. По данным литературы остеопения носит распространенный характер $[6,7,8]$. Поэтому выявление факторов риска и формирование групп риска для своевременной и профилактики остеопороза и восполнения МПК сохраняет актуальность в настоящее время.

\section{ЦЕЛЬ}

Изучить факторы, ассоциированные с развитием остеопении в послеродовом периоде

\section{МЕТОДЫ}

\section{Дизайн исследования}

Проведено наблюдательное сплошное одномоментное одноцентровое исследование с участием 124 женщин, проживающих в Санкт-Петербурге и Ленинградской области.

\section{Критерии соответствия}

К участию в исследование приглашали родильниц в возрасте от 20 до 35 лет на 3-5 сутки после родов при условии добровольного подписания формы информированного согласия.

В исследование не включали женщин:

- после многоплодной беременности;

- с заболеваниями, являющимися причиной вторичного остеопороза;

- принимающих лекарственные препараты, влияющие на костный обмен.

\section{Условия проведения}

Исследование проведено на базе «Национального медицинского исследовательского центра им. В.А. Алмазова» в условиях послеродового отделения.

\section{Продолжительность исследования}

Исследование проведено в период с октября 2013 по ноябрь 2014 года.

\section{Описание медицинского вмешательства}

Участницы исследования заполняли анкеты и сдавали образцы крови - по 10 мл из кубитальной вены в пробирку. Так же всем участницам выполнена рентгеновская остеоденситометрия в трех отделах скелета: дистальном отделе предплечья, проксимальном отделе бедра, поясничном отделе позвоночника.

\section{Основной исход исследования}

Конечной точкой исследования были показатели минеральной плотности костной ткани после родов.

\section{Дополнительные исходы исследования}

В ходе исследования так же оценивали концентрацию витамина D в крови пациенток.

\section{Анализ в подгруппах}

По результатам рентгеновской остеоденситометрии сформированы группы: 1-я - со снижением МПК, соответствующим остепении, и 2 - группа - с нормальной МПК. Для оценки степени снижения МПК применялся Z-критерий. За снижение МПК, соответствующее остеопении, принимали Z-критерий от -1 до -2,5 SD, от -2,5 SD снижение МПК соответствующее остеопорозу.

\section{Методы регистрации исходов}

Анкеты, содержащие вопросы по анамнезу жизни, перенесенным травмам, переломам, вредным привычка, наследственности, наличию хронических заболеваний (хронический пиелонефрит, аутоиммунный тиреоидит, наследственная тромбофилия), учитывался прием лекарственных препаратов, поливитаминов. В рамках анкеты 
была собрана информация о гинекологическом анамнезе (возраст менархе, нарушение менструального цикла по типу опсоменорреи, аменореи, дисфункциональные маточные кровотечения (ДМК)), акушерском анамнезе (количество беременностей и родов, исходы предыдущих беременностей), течении настоящей беременности (угроза прерывания, осложнения беременности - аутоиммуный тиреоидит, гестационный сахарный диабет, преэклампсия). Для оценки степени снижения МПК применялся Z-критерий (критерии остеопении и остеопороза указаны выше). Содержание 25(OH)D >30 нг/мл считали показателем нормальной концентрации витамина D, 25(OH)D <30 нг/мл считали показателем недостаточности витамина D, 25(OH) $\mathrm{D}<20$ нг/мл - показателем дефицита витамина D.

Рентгеновская остеоденситометрия проводилась на остеденситометре Lunar Prodigy DF 110186 в поликлиническом отделении ФГБУ «НМИЦ им. В.А.Алмазова». 25-OH-D определяли хемилюминесцентным методом на анализаторе Architect 2000, концентрацию ПТГ в сыворотке крови определяли с использованием наборов и калибраторов фирмы «Roche Diagnostics» (Германия) методом иммуноферментного анализа на анализатоpe "Cobas E311", Roche (Швейцария) на базе ЦКДЛ ФГБУ «НМИЦ им. В.А. Алмазова» Минздрава России.

\section{Этическая экспертиза}

Этический комитет при ФГБУ «НМИЦ им. В.А.Алмазова», протокол № 194 от 12.06.13 одобрил клиническое исследование в рамках выполнения диссертационный работы.

\section{Статистический анализ}

Размер выборки предварительно не рассчитывался. Статистическую обработку результатов проводили с помощью программы STATISTICA 10 (StatSoft, Inc.). Количественные показатели представлены в виде Среднее \pm Стандртное Отклонение $(\mathrm{M} \pm \mathrm{SD})$. Критический уровень значимости различия показателей принимали равным 0,05. Для анализа групп по таблицам сопряженности дихотомических признаков применялся точный критерий Фишера. Для сравнения групп по количественным или порядковым признакам был использован однофакторный дисперсионный анализ (ANOVA) и F-критерии Фишера или Крускала-Уоллиса, соответственно.

\section{РЕЗУЛЬТАТЫ}

Объекты (участники) исследования

В исследование включено 112 родильниц. Средний возраст обследуемых составил в 1-ой группе 28,3 22,4 лет, во 2-ой группе 30,4 2,8 лет. Индекс массы тела (ИМТ)

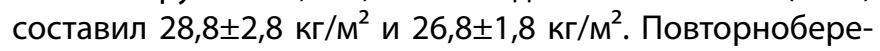
менные, повторнородящие составили 52\% обследуемых в 1-ой группе и 65\% - во 2-ой группе. Группы по данным признакам однородные и достоверных различий между группами нет.

\section{Основные результаты исследования}

По результатам рентгеновской остеоденситометрии сформировано две группы: 1- группу $(\mathrm{N}=70)$ составили родильницы со снижением МПК, соответствующим остеопении, 2 - группу ( $\mathrm{N}=42)$ родильницы с нормальной МПК (таб. 1).

Результаты остеоденситометрии, отражающие МПК в группах наблюдения представлены на рисунке 1. Снижение МПК, соответствующее остеопении, в дистальном отделе предплечья встречалось у $48 \%$ обследуемых (Z-критерий $-2,2 \pm 0,28 \mathrm{SD})$, в поясничном отделе позвоночника

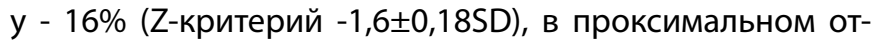

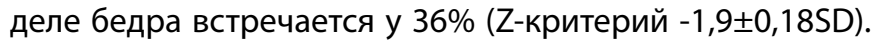
Снижение МПК, соответствующее остеопорозу, встречалось В дистальном отделе предплечья у $22 \%$ (Z-критерий $-3,3 \pm 0,18 \mathrm{SD})$, в проксимальном отделе бедра у 2\% (Z-критерий $-2,8 \pm 0,18 \mathrm{SD})$ родильниц.

При оценке анамнестических данных: только в 1-ой группе в анамнезе встречались переломы у ближайших родственников до 50 лет - у 2 \% обследуемых и у 7\% женщин - переломы в анамнезе в подростковом возрасте, у двух родильниц случились переломы во время течения настоящей беременности. Нельзя исключить генетический фактор влияния МПК у данных женщин. Курение отмечено у 1 женщины в 1-ой группе. В 1-ой группе хронические заболевания (аутоиммунный тиреоидит, хронический пиелонефрит) встречались у 23 \%, во 2 -ой группе - у 19\% обследованных. Женщинам не требовалось назначение специфической терапии, поэтому значимого вклада хронических заболеваний во влиянии на МПК в данном исследовании не ожидалось. Оценка характера питания выявила следующее: в 1-ой группе у 92\% родильниц потребление в питании кальция содержащий было менее 800 мг/сутки, во 2- ой группе - у 75\%. Установлена взаимосвязь показателей МПК с низкокальциевым питанием (менее 800 мг/сутки, р<0,041). При анализе антропометрических данных: дефицит массы тела в подростковом возрасте встречался только в 1-ой группе у $11 \%$ обследуемых ( $<<0,013)$. Избыточная масса тела в 1 -ой группе у $60 \%$, во 2 -ой группе - у $47 \%$ родильниц. Установлена значимость фактора ИМТ $(p<0,018)$ на снижение МПК в группах наблюдения.

Таблица 1.Сравнительная характеристика групп пациенток

\begin{tabular}{|c|c|c|}
\hline Сравниваемый параметр & 1-ая группа $(\mathrm{N}=70)$ & 2-ая группа ( $N=42)$ \\
\hline 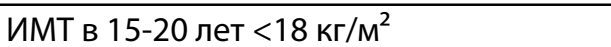 & $8(11 \%)$ & - \\
\hline ИМТ $\geq 25 \mathrm{kr} / \mathrm{M}^{2}$ & $42(60 \%)$ & $22(47 \%)$ \\
\hline $\begin{array}{l}\text { Хронические заболевания (пиелонеф- } \\
\text { рит,тиреоидит) }\end{array}$ & $16(23 \%)$ & $8(19 \%)$ \\
\hline Употребление кальция <800 мг/сут & $65(92 \%)$ & $32(75 \%)$ \\
\hline Витамин D >30 нг/мл (норма) & - & $6(14 \%)$ \\
\hline Витамин D <30 нг/мл (недостаточность) & $12(18 \%)$ & $29(70 \%)$ \\
\hline Витамин D <20 нг/мл (дефицит) & $58(82)$ & $7(16 \%)$ \\
\hline
\end{tabular}




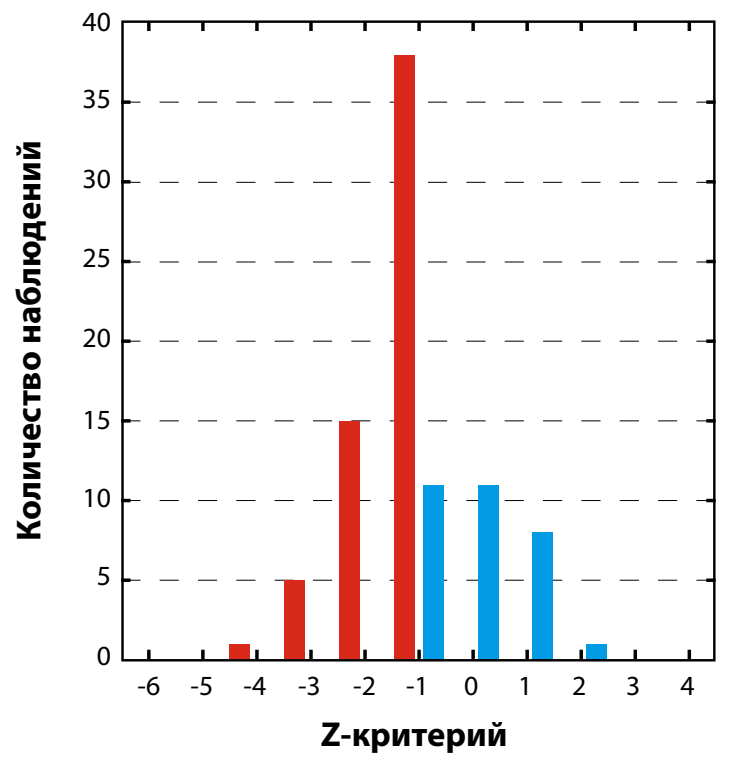

Группа с нормальной МПК Группа со нснижением МПК

Рис. 1. Распределение МПК в группах наблюдения.

Оценка акушерско-гинекологического анамнеза и течения беременности показала следующее (таб. 2): средний возраст менархе составил в 1-ой группе 13,4 2,4 лет,

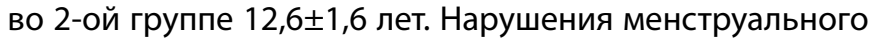
цикла (НМЦ) встречались в 1-ой группе у 12\%: по типу олигоменнореи - у 6\%, по типу опсоменорреии - 4\%, аменорея - у 2\%; во 2-ой группе у 5 \%: по типу олигоменорреии 2\%, ДМК 2\%. Следует отметить статистически значимую связь фактора нарушения менструального цикла на МПК в поясничном отделе позвоночника в группе с остеопенией $(p<0,052)$. Гормонозаместительную терапию не получала ни одна из женщин, комбинированные оральные контрацептивы получали 7\% в 1-ой группе и 5\% во 2-ой группе. Беременность у всех женщин наступила самостоятельно. Повторнобеременные, повторнородящие составили 52\% обследуемых в 1-ой группе и 65\% - во 2-ой группе. Значимой связи влияния паритета (количества и частоты родов) на МПК установлено не было. Течение беременности: в 1-ой группе угроза прерывания беременности на ранних сроках отмечалась у $10 \%$ женщин, во 2-ой группе - у 8\%. Гестационный сахарный диабет встре-

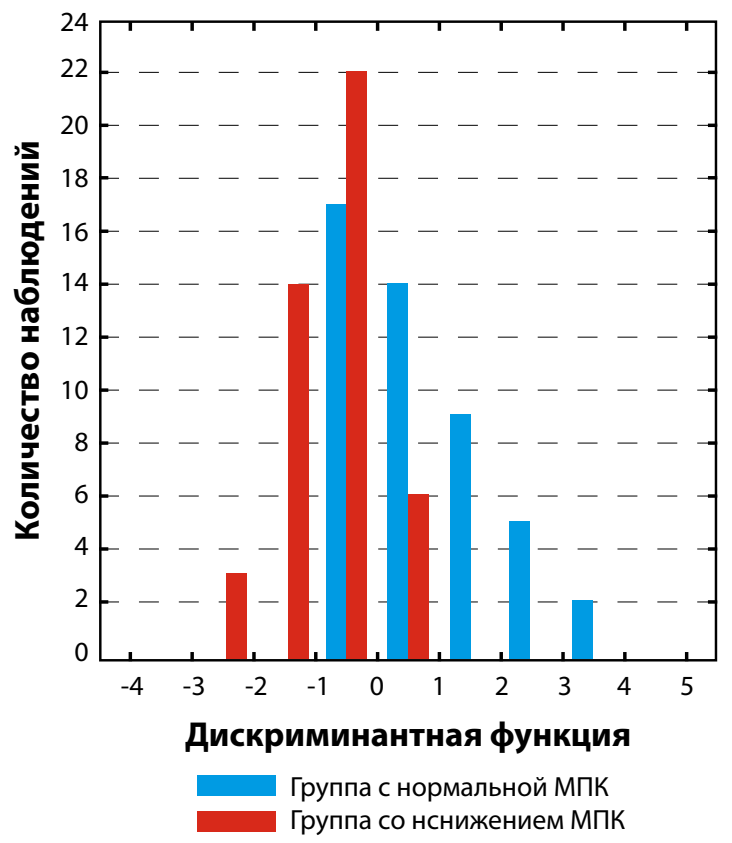

Рис. 2. Гистограмма дискриминантной функции характеризует относительный риск развития остеопении.

чался у $26 \%$ в 1-ой группе и у $20 \%$ во 2-ой группе. Аутоиммунный тиреоидит в 1-ой группе у $12 \%$, во 2-ой группе - у 14\%. Преэклампсия встречалась только в первой группе, у 12\% родильниц. Установлена взаимосвязь между МПК в дистальном отделе предплечья и преэклапсией $(p<0,042)$, что требует дальнейшего изучения. При анализе лабораторных данных: среднее значение 25-(OH)-D

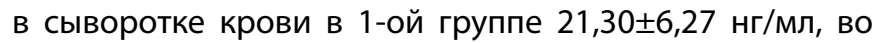
2-ой группе - 21,67士4,4 нг/мл, что соответствует недостаточности витамина D в обеих группах. Все беременные получали поливитаминные комплексы, содержащие стандартную дозу 400 ME витамина D. Была проведена оценка распространенности дефицита и недостаточности витамина D: у всех родильниц в 1- ой группе и у $86 \%$ во 2-ой группе. Выявлена значимость влияния снижения $25-\mathrm{OH}-\mathrm{D}<25$ нг/мл на МПК ( $<<0,021)$.Уровень ПТГ в груп-

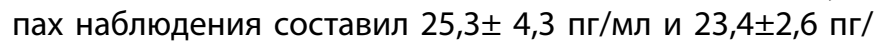
мл соответственно, что в пределах референсных значений. Учитывая выявленные факторы снижения МПК, мы оценили их совместное взаимодействие и получили следующее: сочетание факторов таких как ИМТ в 15-20 лет

Таблица 2. Данные акушерско-гинекологического анамнеза и сопутствующих заболеваний в исследуемых группах

\begin{tabular}{lcc}
\hline \multicolumn{1}{c}{ Сравниваемый параметр } & 1-ая группа (N=70) & 2-ая группа (N=42) \\
\hline Средний возраст менархе, лет & $13,4 \pm 2,4$ & $12,6 \pm 1,6$ \\
НМЦ & - & $1(2 \%)$ \\
Олигоменнорея & $4(6,00 \%)$ & - \\
Опсоменоррея & $3(4 \%)$ & $1(2 \%)$ \\
Аменоррея & $1(2 \%)$ & $1(2 \%)$ \\
ДМК & - & $26(65 \%)$ \\
Угороза прерывния & $7(10 \%)$ & $8(20 \%)$ \\
Повторнобеременные,повторнородя- \\
щие \\
Гестационный сахарный диабет & $36(52 \%)$ & - \\
Преэклампсия & $18(26 \%)$ & $6(14 \%)$ \\
АИТ & $8(12 \%)$ & $8(12 \%)$ \\
\hline
\end{tabular}




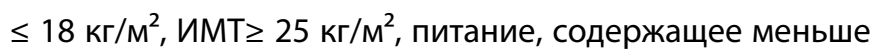
800 мг кальция в сутки, увеличивают риск снижения МПК в 11 раз в дистальном отделе предплечья, $\mathrm{OR}=11,47059$; Cl 95\% = [4,0326; 32,627]

На рисунке 2 представлена гистограмма построенной дискриминантной функции.

\section{Дополнительные результаты исследования}

Выявлен распространенный дефицит и недостаточность витамина D. Дефицит витамина D выявлен у $82 \%$ родильниц в 1 группе у 18\% выявлена недостаточность витамина D; во 2 группе дефицит витамина D встречался у $16 \%$ родильниц ,недостаточность витамина D встречалась у $70 \%$, у $14 \%$ нормальные значения витамина D

\section{Нежелательные явления}

При проведении анкетирования, рентгеновской остеоденситометрии, забора образцов крови нежелательные явления не зарегистрированы.

\section{ОБСУЖДЕНИЕ}

\section{Резюме основного результата исследования}

Наиболее существенное влияние на развитие остеопении у родильниц оказывают следующие факторы снижения МПК: индекс массы тела $\leq 18$ кг/м²; уровень 25-OH-D <25 нг/мл; питание с содержанием кальция $<800$ мг в сутки, преэклампсия. Совокупность этих факторов существенно может повышать риск развития остеопении в дистальном отделе предплечья.

\section{Обсуждение основного результата исследования}

При анализе анамнестических факторов риска нами отмечен значимый фактор питания с низким содержанием кальция и витамина D, что согласуется с данными зарубежных авторов. По нашим данным 80-87\% имеют недостаточное потребление кальция, что сказывается на состоянии фосфорно-кальциевого обмена. Однако снижение МПК у родильниц в основной группе, как показало наше исследование, может быть связано с влиянием ряда других факторов, наряду с несбалансированным питанием и вероятно низким МПК до наступления беременности.

Работы, посвященные изучению образа питания немногочисленны. По данным польских авторов, проводивших исследования у женщин 20-30 лет только 25\% имеют достаточное потребление кальция в сутки и только 10\% женщин дополнительно принимают добавки кальция и витамина D. По данным европейских исследований частота недостаточного потребления витамина D у взрослых женщин составляет 77-100 \% и недостаточное потребление кальция встречается у 26-61\% в большинстве европейских стран [9]. В работе Д.С. Судакова отмечена взаимосвязь между потреблением кальция и остеопенией: остеопения встречалась в 1,5 раза чаще при потреблении менее 800 мг кальция в сутки [10].

В настоящем исследовании выявлен такой значимый фактор риска снижения МПК, как ИМТ. В обеих группах были женщины с ИМТ соответствующим избыточной массе тела, однако снижение МПК, соответствующее остеопении развивается, вероятно, в связи с сочетанным действием нескольких факторов, влияющих на МПК. По данным ряда авторов, МПК зависит от массы тела и ИМТ. ИМТ в подростковом возрасте может отразиться на пике формирования костной массы, что впоследствии может сказаться на МПК в репродуктивном возрасте. Так, по данным Шишковой подростки с ожирением могут не достигать пика костной массы, что увеличивает последующий риск переломов во взрослом возрасте [11]. Однако в настоящем исследовании, ожирение в подростковом возрасте по анамнестическим данным не встречалось, а дефицит массы тела выявлен только в 1 группе, у 11 пациенток. Была выявлена взаимосвязь между дефицитом массы тела в подростковом возрасте и МПК у родильниц, что вероятно связано с нарушением формирования пика костной массы [13].

По данным Garrett-Mayer масса тела влияет на минерализацию кости во время беременности, так по его данным более высокий ИМТ был связан с большей потерей МПК шейки бедренной кости во время беременности [2], но мы не отметили данной связи в нашем исследовании.

Особенно следует отметить в нашей работе распространенность недостаточности и дефицита витамина D носит практически тотальный характер и достигает 82-92\% всех обследованных родильниц г. Санкт-Петербурга. Однако, развилась остеопения у определенной категории женщин, вероятно в сочетании с другими факторами и низким МПК до наступления беременности. Крупные эпидемиологические исследования подтверждают, что концентрация 25 -(OH)-D ниже определенного порога (30 нмоль/л или 12 нг/мл) связана с уменьшением МПК. В данной работе мы не определяли пороговое значение 25-(OH)-D. Дефицит и недостаточность витамина D является значимым фактором риска снижения МПК, особенно в дистальном отделе предплечья. Можем предположить, что дефицит и недостаточность витамина D приводит к увеличению уровня ПТГ, вторичному гиперпаратиреозу, который поддерживает нормальный уровень кальция за счет мобилизации его из скелета. ПтГ повышает активность остеокластов, и тем самым приводит к снижению МПК преимущественно из дистального отдела предплечья. Однако, уровни ПтГ являются низкими или нормальными для большей части беременных, и поэтому понятие «физиологический гиперпаратиреоз беременности» является дискутабельным. Однако, нами выявлена статистически значимая связь между уровнем 25-(OH)-D, ПТГ, ИМТ и снижением МПК в дистальном отделе предплечья.

В большинстве рандомизированных исследований нарушение менструального цикла, по типу аменореи и опсоменорреи связаны с более низкой МПК в поясничном отделе позвоночника $[15,16]$. В нашем исследовании получены данные о возможном влиянии НМЦ на МПК в поясничном отделе позвоночника, однако ограничения нашего исследования связаны с небольшой распространенностью остеопении в поясничном отделе позвоночника и немногочисленными данными о нарушениях менструального цикла. Так же хотелось бы отметить, что во время беременности, по-видимому, плацентарной синтез эстрогенов, оказывает дополнительное протективное влияние на костный обмен.

Ряд авторов, отмечает, что частые роды и длительный период лактации являются протективными факторами перелома шейки бедра. В некоторых исследованиях выявлено прямое влияние числа рожденных детей на риск 
перелома шейки бедра [4,14]. Однако, ретроспективные эпидемиологические исследования не обнаружили влияния паритета на риск остеопороза или переломов в более позднем возрасте[16,17]. Вероятно, беременность может быть связана с остеопорозом и переломами, особенно если женщина «вступает» в беременность с низкой пиковой костной массой. В нашем исследовании участвовали 50-60\% повторнобеременных, повторнородящих, однако связи между паритетом и снижением МПК не была установлено, что, вероятно, связано с достаточными интервалами между беременностями и родами, длительностью лактации и аменореи меньше 6 месяцев. Так же мы не оценивали МПК после первых родов и до наступления беременности.

Таким образом, несмотря на распространенность дефицита и недостаточности витамина D, снижение МПК встречается у $60 \%$ обследуемых, что связано с сочетанием факторов риска снижения МПК, таких как ИМТ в 15-20 лет $\leq 18$ кг/м²; питание, содержащее меньше 800 мг/сутки кальция, нарушение менструального цикла увеличивают риск снижения МПК в дистальном отделе предплечья в 11 раз при беременности. Так же мы предполагаем, что некоторые случаи остеопороза при беременности могут представлять собой идиопатический остеопороз, встречающийся у беременных женщин. Нельзя так же исключить генетический фактор, так при оценке анамнестических данных было указание на переломы у близких родственников и переломы случились у самих женщин во время беременности.

\section{Ограничения исследования}

Возможные ограничения исследования связаны с недостаточной выборкой пациенток, отсутствием данных денситометрии до беременности, особенно у женщин с
НМЦ в репродуктивном периоде. Данные ограничения не позволяют оценить динамику снижения МПК, возникающую у родильниц.

\section{ЗАКЛЮЧЕНИЕ}

Учитывая высокую распространенность остеопении на фоне недостаточности и дефицита витамина D, можно рекомендовать прием препаратов кальция и витамина D согласно международным рекомендациям на этапе планирования беременности, в период беременности и лактации, а также выполнение остеоденситометрии после родов у родильниц из группы риска по снижению МПК. В настоящем исследовании выявлены следующие возможные факторы риска снижения МПК у родильниц: ИМТ в 15-20 лет $\leq 18$ кг/м², ИМТ на момент исследования $\geq 25 \mathrm{kг} / \mathrm{M}^{2}$; концентрация 25-OH-D <25 нг/мл; питание с содержанием кальция меньше 800 мг в сутки; преэклампсия, нарушение менструального цикла. В группе с сочетанием указанных факторов остеопения в дистальном отделе предплечья встречается в 11 раз чаще. Дистальный отдел предплечья может служить маркером снижения МПК в период беременности.

\section{ДОПОЛНИТЕЛЬНАЯ ИНФОРМАЦИЯ.}

Источник финансирования. Исследование проведено при поддержке ФГБУ нМИЦ им. В.А. Алмазова.

Конфликт интересов. Авторы декларируют отсутствие явных и потенциальных конфликтов интересов, связанных с публикацией настоящей статьи.

Участие авторов. Все авторы внесли значимый вклад в проведение исследования, анализ результатов и подготовку статьи, прочли и одобрили финальную версию текста перед публикацией.

\section{СПИСОК ЛИТЕРАТУРЫ | REFERENCES}

1. Singh R, Gupta S, Awasthi A. Differential effect of predictors of bone mineral density and hip geometry in postmenopausal women: a cross-sectional study. Archives of Osteoporosis. 2015;10(1). doi: 10.1007/s11657-015-0246-z.

2. Wei W, Shary JR, Garrett-Mayer E, et al. Bone mineral density during pregnancy in women participating in a randomized controlled trial of vitamin D supplementation. The American Journal of Clinical Nutrition. 2017;106(6):1422-1430. doi: 10.3945/ajcn.116.140459.

3. Møller UK, við Streym S, Mosekilde L, Rejnmark L. Changes in bone mineral density and body composition during pregnancy and postpartum. A controlled cohort study. Osteoporos. Int. 2011;23(4):12131223. doi: 10.1007/s00198-011-1654-6.

4. El Maghraoui A, Do Santos Zounon AA, Jroundi I, et al. Reproducibility of bone mineral density measurements using dual X-ray absorptiometry in daily clinical practice. Osteoporos. Int. 2005;16(12):17421748. doi: 10.1007/s00198-005-1916-2.

5. Namgung R, Tsang RC. Bone in the pregnant mother and newborn at birth. Clin. Chim. Acta. 2003;333(1):1-11. doi: 10.1016/s00098981(02)00025-6.

6. Di Gregorio S, Danilowicz K, Rubin Z, Mautalen C. Osteoporosis with vertebral fractures associated with pregnancy and lactation. Nutrition. 2000;16(11-12):1052-1055. doi: 10.1016/s0899-9007(00)00430-5.

7. Kovacs CS, Kronenberg HM. Maternal-Fetal Calcium and Bone Metabolism During Pregnancy, Puerperium, and Lactation1. Endocr. Rev. 1997;18(6):832-872. doi: 10.1210/edrv.18.6.0319.

8. Yun KY, Han SE, Kim SC, et al. Pregnancy-related osteoporosis and spinal fractures. Obstetrics \& Gynecology Science. 2017;60(1):133. doi: 10.5468/ogs.2017.60.1.133.
9. Sidor P, Glabska D, Wlodarek D. Analysis of the dietary factors contributing to the future osteoporosis risk in young Polish women. Rocz. Panstw. Zakl. Hig. 2016;67(3):279-285

10. Судаков Д.С. Оценка кальций-фосфорного обмена и ремоделирования костной ткани у женщин при физиологической беременности: Дис. ... канд. мед. наук. -СанктПетербург; 2011. [Sudakov DS. Otsenka kal'tsiy-fosfornogo obmena i remodelirovaniya kostnoy tkani u zhenshchin prifiziologicheskoy beremennosti. [dissertation] Saint Petersburg; 2011. (in Russ)].

11. Шишкова В.Н. Ожирение и остеопороз // Остеопороз и остеопатии. - 2011. - Т. 14. - №1. - С. 21-26. [Shishkova VN, Shishkova VN. Obesity and osteoporosis. Osteoporosis and bone diseases. 2011;14(1):21-26. (in Russ).] doi: 10.14341/osteo2011121-26.

12. Wiksten-Almströmer M, Hirschberg AL, Hagenfeldt K. Prospective follow-up of menstrual disorders in adolescence and prognostic factors. Acta Obstet. Gynecol. Scand. 2008;87(11):1162-1168. doi: 10.1080/00016340802478166.

13. Balasuriya CND, Evensen KAl, Mosti MP, et al. Peak Bone Mass and Bone Microarchitecture in Adults Born With Low Birth Weight Preterm or at Term: A Cohort Study. J. Clin. Endocr. Metab. 2017;102(7):2491-2500. doi: 10.1210/jc.2016-3827.

14. Laskey MA, Price RI, Khoo BCC, Prentice A. Proximal femur structural geometry changes during and following lactation. Bone. 2011;48(4):755-759. doi: 10.1016/j.bone.2010.11.016.

15. Meczekalski B, Katulski K, Czyzyk A, et al. Functional hypothalamic amenorrhea and its influence on women's health. J. Endocrinol. Invest. 2014;37(11):1049-1056. doi: 10.1007/s40618-014-0169-3.

16. Ward KA, Adams JE, Mughal MZ. Bone status during adolescence, 
pregnancy and lactation. Curr. Opin. Obstet. Gynecol. 2005;17(4):435439. doi: 10.1097/01.gco.0000175365.65835.ad.

17. Gajic-Veljanoski O, Papaioannou A, Kennedy C, et al. Osteoporotic fractures and obesity affect frailty progression: a longitudinal analysis of the Canadian multicentre osteoporosis study. BMC Geriatr. 2018;18(1). doi: 10.1186/s12877-017-0692-0.

18. Chin K-Y, Low N, Dewiputri W, Ima-Nirwanaa S. Factors Associated with Bone Health in Malaysian Middle-Aged and Elderly Women Assessed via Quantitative Ultrasound. Int. J. Env. Res. Public Health. 2017;14(7):736. doi: 10.3390/ijerph14070736.

19. El Maghraoui A, Guerboub AA, Mounach A, et al. Body mass index and gynecological factors as determinants of bone mass in healthy Moroccan women. Maturitas. 2007;56(4):375-382. doi: 10.1016/j. maturitas.2006.10.004.

\section{ИНФОРМАЦИЯ ОБ АВТОРАХ [AUTHORS INFO]}

Новикова Татьяна Васильевна [Tatiana V. Novikova]; адрес: Россия, 197341 Санкт-Петербург, улица Аккуратова, д. 2 [address: 2 Akkuratov street 197341, Saint Petersbur, Russia]; ORCID: https://orcid.org/0000-0001-8758-6857; eLibrary SPIN: 7143-2088; e-mail: tanyanovikova.85@mail.ru.

Кузнецова Любовь Владимировна, к.м.н. [Lubov V. Kuznetsova, MD, PhD]; ORCID: https:// orcid.org/0000-0002-1453-2118; eLibrary SPIN: 5355-0262; e-mail: krivo73@mail.ru

Яковлева Наталья Юрьевна [Natalia Yu. Yakovleva]; ORCID: http://orcid.org/0000-0002-0207-1035; eLibrary SPIN: 38069335; e-mail:Natalis.1986@mail.ru

Зазерская Ирина Евгеньевна, д.м.н. [Irina E. Zazerskaya, MD, PhD]; ORCID: https://orcid.org/0000-0003-4431-3917; eLibrary SPIN: 5683-6741; e-mail: zazera@mail.ru

\section{ЦИТИРОВАТЬ:}

Новикова Т.В., Кузнецова Л.В., Яковлева Н.Ю., Зазерская И.Е. Факторы, ассоциированные со снижением МПК в послеродовом периоде. // Остеопороз и остеопатии. — 2018. — Т. 21. — №1. - C.10-16. doi: 10.14341/osteo9653

\section{TO CITE THIS ARTICLE:}

Novikova TV, Kuznetsova LV, Yakovleva NYu, Zazerskaya IE. Factors influencing bone mineral density in postpartum women. Osteoporosis and bone diseases. 2018;21(1):10-16. doi: 10.14341/osteo9653 